\title{
Towards Understanding the Workspace of The Upper Extremities
}

\author{
Karim Abdel-Malek ${ }^{1}$, Ph.D. \\ Jingzhou Yang ${ }^{1}$, M.S. \\ Richard Brand ${ }^{2}$, M.D. \\ Michael Vannier ${ }^{3}$, M.D. \\ ${ }^{1}$ Department of Mechanical Engineering \\ ${ }^{2}$ Department of Orthopedics \\ ${ }^{3}$ Department of Radiology \\ THE UNIVERSITY OF IOWA \\ lowa City, IA 52242
}

\section{Abstract}

Significant attention in recent years has been given towards obtaining a better understanding of human joint ranges, measurement, and functionality, especially in conjunction with commands issued by the central nervous system. Studies of those commands often include computer algorithms to describe path trajectories. These are typically in "open-form" with specific descriptions of motions, but not "closed form" mathematical solutions of the full range of possibilities. This paper proposes a rigorous "closed form" kinematic formulation to model human limbs, understand their workspace, and delineate barriers therein where a path becomes difficult or impossible owing to physical constraints. The novel ability to visualize barriers in the workspace emphasizes the power of these closed form equations. Moreover, this formulation takes into account joint limits in terms of ranges of motion. Examples include the workspaces of a typical forearm, a typical finger, and is used to illustrate the visualization of the progress in the functionality of a wrist undergoing rehabilitation.

Keywords: Limb workspace, ergonomics, ranges of motion, shoulder, wrist, arm, anthropometry. 


\section{Introduction}

The ability to define specific work limitations, physical impairment, the changes in limitations with injury or disease, and the improvements with therapy have always been problematic in part owing to their subjective nature. Various authors proposed more objective measures including specific limitations of motions (Van Thiel, et al. 1999, Kawato 1996, Kawato and Wolpert 1998, Uno, et al 1989, Cruse and Bruwer 1987 and Bruwer and Cruse 1990) on which impairment is often largely based (e.g., AMA Guides to the Evaluation of Permanent Impairment [AMA 1997]). The reader is also referred to the recent comparison of commercially available measuring systems (Richards 1998). However, in a given patient, none of these approaches can identify the entire range of limitations given specific constraints in particular directions. Consider tracking of point on a finger in space. The volume generated by every possible point touched by this finger is called the workspace of that limb. Complete identification of workspace of that finger is important to:

(1) Understand neural strategies allowing the positioning and orienting of the hand during voluntary reaching movements, especially that of the human upper extremity.

(2) Quantify the full functional potential of a joint.

(3) Select ergonomic postures reducing stresses induced in adjacent joints

The process of moving the hand to a target in space involves a series of sensorimotor transformations that converts sensory signal of visual data about the location and orientation of the target object (and the arm) into a set of motor commands that will bring the hand to the desired position. The CNS learns and maintains internal models of these sensorimotor transformations such as plane or curved trajectories (Klein-Breler, et al. 1998) and horizontal 
plane trajectories (Suzuki, et al. 1997). While various models simulate this task (for example the commercial code JACK [Badler, 1997]), most of these models rely upon experimental data, or incomplete (i.e., "open-form") numerical simulations. These simulations describe specific situations, but owing to lack of formal description (i.e., "closed form" simulations) cannot simulate the entire range of possibilities.

Impaired arms exhibit well-defined workspace deficits (Reinkensmeyer, et al. 1999). This suggests range of motion and constraint forces may prove useful for precise monitoring of arm impairment and the effects of treatment techniques targeted at abnormal synergies and workspace deficits (Yu and Donaldson 1999, Johnson, et al. 1999, Kirstukas, et al. 1992). These results were consistent with the standard flexion and extension "synergies" described in the clinical literature. Indeed, this type of approach has been used for the evaluation of arm impairment after brain injury (Reinkensmeyer, et al. 1999). Studies of limitations of joint rotation on the independence of hand rotation (Kamper and Zev-Rymer 1999 and Schillings, et al. 1998), while contributing to the discussion of limb workspace and ergonomics have been limited by the number of degrees of freedom modeled and by the numerical algorithms used to generate the workspace.

A comprehensive human model using the Visible Human Dataset (VHD) for the European project CHARM demonstrates the need for a better understanding of the workspace of human limbs (Kalra, et al. 1995, Maurel and Thalamann 1999, Maurel and Thalamann 2000 and Maurel, et al. 1996). Although this research group has addressed the topological aspects of limbs and joints, the generation of the workspace has not been demonstrated. 
The understanding of trajectory formations inside the workspace of human limbs is, to a great extent, dependent upon the identification of control barriers that exist as impediments to motion and that may hinder the execution of a planned trajectory. A rigorous mathematical formulation based on kinematics will first be introduced. Because of this formulation, we will show that barriers inside the workspace are identified. More importantly, closed form equations of the workspace will be established. As a result, a method for quantifying the mobility (functionality) of a joint will be demonstrated to the wrist. Furthermore, it will be shown that visualization of the internal of the workspace provides a powerful tool for grasping the limitations.

\section{Modeling and Formulation}

Whereas the anatomy of limbs and their joints are indeed very complex (as evidenced by the debate in the literature on the correct method for modeling joint motion), we will employ a kinematic pair (or combination thereof) as used in the field of robotics. For example, if the

resultant motion is rotational, the joint will be modeled as a revolute joint. The effect of a spherical joint is modeled as three revolute joints whose axes intersect at the center of the sphere. Indeed, all anatomical joints can be modeled using basic kinematic pairs (any joint). The elbow joint is modeled as a revolute joint, where $q_{1}$ is the joint variable (Figure 1). 


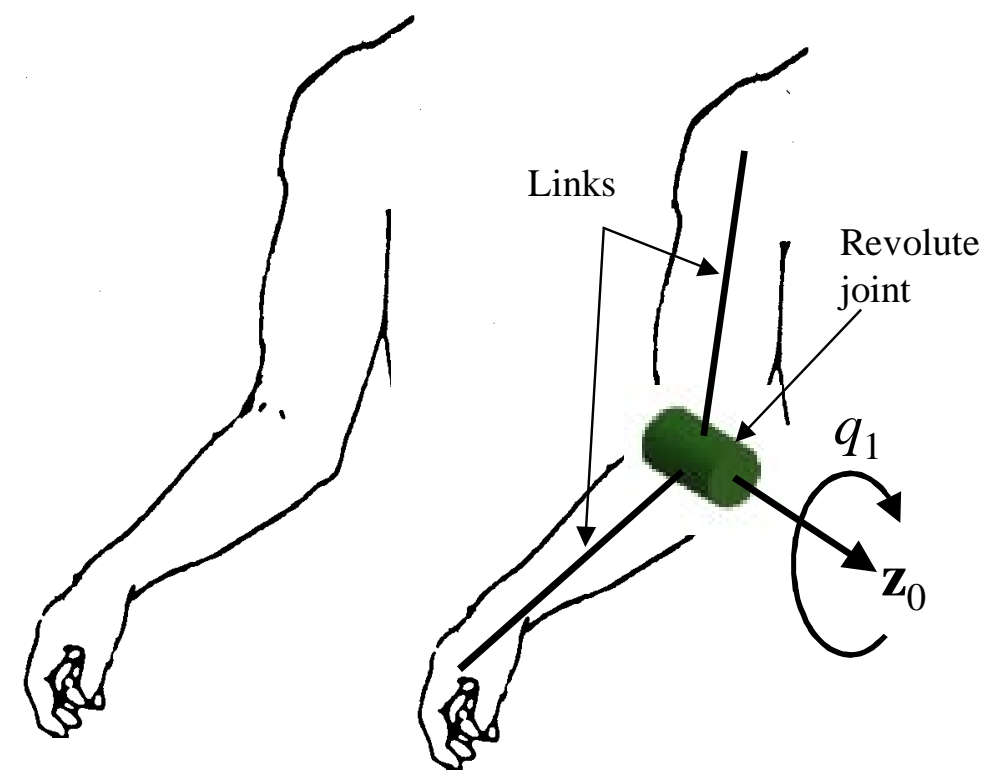

Fig. 1 Definition of a kinematic pair (e.g., an elbow is modeled as a revolute joint)

In order to obtain a systematic representation of any serial kinematic chain, we define $\mathbf{q}^{*}=\left[\begin{array}{lll}q_{1} & \ldots & q_{n}\end{array}\right]^{T} \in \mathbf{R}^{n}$ as the vector of $n$-generalized coordinates defining the motion of a limb with respect to another, where $q_{i}$ are the individual joint variables. The position vector function (shown in Fig. 2) generated by a point of interest written as a multiplication of rotation matrices and position vectors is expressed by

$$
\mathbf{x}=\boldsymbol{\xi}\left(\mathbf{q}^{*}\right)=\left[\begin{array}{c}
x\left(\mathbf{q}^{*}\right) \\
y\left(\mathbf{q}^{*}\right) \\
z\left(\mathbf{q}^{*}\right)
\end{array}\right]=\sum_{i=1}^{i=n}\left[\prod_{j=1}^{j=i-1}{ }^{j-1} \mathbf{R}_{j}\right]^{i-1} \mathbf{p}_{i}
$$

where $\mathbf{x}=\left[\begin{array}{lll}x & y & z\end{array}\right]^{T}$ and both ${ }^{i} \mathbf{p}_{j}$ and ${ }^{i} \mathbf{R}_{j}$ are defined using the Denavit-Hartenberg (D-H) representation method (Denavit and Hartenberg 1955, Paul 1981, and Fu et al. 1987) such that

$$
{ }^{i-1} \mathbf{R}_{i}=\left[\begin{array}{ccc}
\cos q_{i} & -\cos \alpha_{i} \sin q_{i} & \sin \alpha_{i} \sin q_{i} \\
\sin q_{i} & \cos \alpha_{i} \cos q_{i} & -\sin \alpha_{i} \cos q_{i} \\
0 & \sin \alpha_{i} & \cos \alpha_{i}
\end{array}\right] \text { and }{ }^{(i-1)} \mathbf{p}_{i}=\left[\begin{array}{lll}
a_{i} \cos q_{i} & a_{i} \sin q_{i} & d_{i}
\end{array}\right]^{T}
$$


where $q_{i}$ is the joint angle from $\mathbf{x}_{i-1}$ axis to the $\mathbf{x}_{i}$ axis, $d_{i}$ is the shortest distance between $\mathbf{x}_{i-1}$ and $\mathbf{x}_{i}$ axes, $a_{i}$ is the offset distance between $\mathbf{z}_{i}$ and $\mathbf{z}_{i-1}$ axes, and $\alpha_{i}$ is the offset angle from $\mathbf{z}_{i-1}$ and $\mathbf{z}_{i}$ axes.

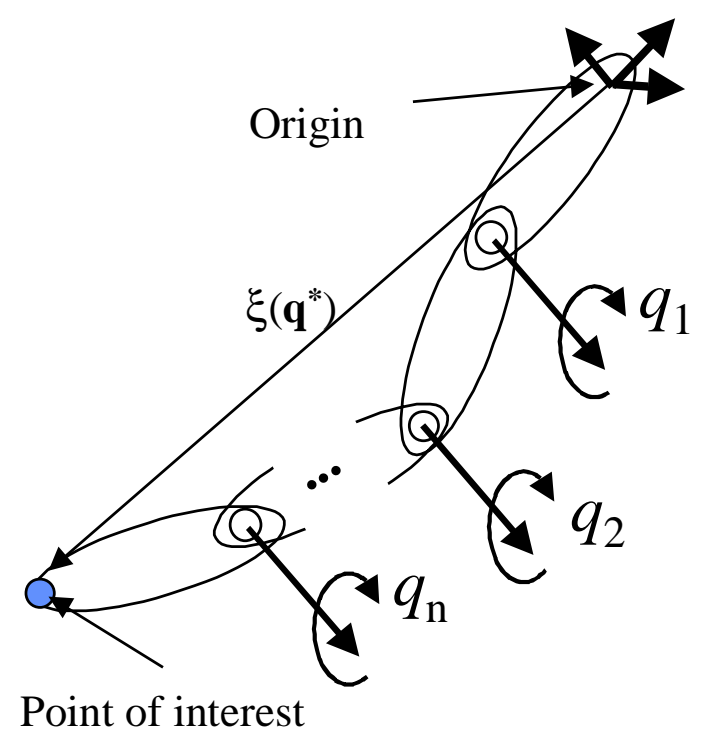

Fig. 2 Definition of the position vector function $\boldsymbol{\xi}\left(\mathbf{q}^{*}\right)$

The vector function $\xi\left(\mathbf{q}^{*}\right)$ characterizes the set of all points touched by the point of interest. The aim is to determine the envelope of this set (also sometimes called the "reach envelope" Molenbroek 1998 and $\mathrm{Li}$ and Xi 1990) At a specified position in space given by $\left(x_{p}, y_{p}, z_{p}\right)$, Eq. 1 can be written as a constraint function as

$$
\boldsymbol{\Omega}\left(\mathbf{q}^{*}\right)=\left[\begin{array}{c}
x\left(\mathbf{q}^{*}\right)-x_{p} \\
y\left(\mathbf{q}^{*}\right)-y_{p} \\
z\left(\mathbf{q}^{*}\right)-z_{p}
\end{array}\right]=\mathbf{0}
$$

In mathematical terms, the expression defined by Eq. (3) is indeed a Manifold with boundary and cannot readily be visualized. 
Joint limits (ranges of motion) are imposed in terms of inequality constraints in the form of

$$
q_{i}^{L} \leq q_{i} \leq q_{i}^{U}
$$

where $i=1, \ldots n$. We transform the inequalities above into equalities by introducing a new set of generalized coordinates $\lambda=\left[\begin{array}{lll}\lambda_{1} & \ldots & \lambda_{n}\end{array}\right]^{T}$ such that

$$
q_{i}=\left(\left(q_{i}^{L}+q_{i}^{U}\right) / 2\right)+\left(\left(q_{i}^{U}-q_{i}^{L}\right) / 2\right) \sin \lambda_{i} \quad i=1, \ldots, n
$$

In order to include the effect of joint limits, it is proposed to augment the constraint equation $\Omega\left(\mathbf{q}^{*}\right)$ with the parametrized inequality constraints of Eq. (5) such that

$$
\mathbf{H}(\mathbf{q})=\left[\begin{array}{c}
x\left(\mathbf{q}^{*}\right)-x_{p} \\
y\left(\mathbf{q}^{*}\right)-y_{p} \\
z\left(\mathbf{q}^{*}\right)-z_{p} \\
q_{i}-a_{i}-b_{i} \sin \lambda_{i}
\end{array}\right]=\mathbf{0} \quad i=1, \ldots n
$$

where $\quad \mathbf{q}=\left[\begin{array}{ll}\mathbf{q}^{* T} & \lambda^{T}\end{array}\right]^{T}$ is the vector of all generalized coordinates. Note that although $n-$ new variables $\left(\lambda_{i}\right)$ have been added, $n$-equations have also been added to the constraint vector function without loosing the dimensionality of the problem.

The Jacobian (named after the German Mathematician Carl G. Jacobi) of the constraint function $\mathbf{H}(\mathbf{q})$ at a point $\mathbf{q}^{0}$ is the $(3+n) \times 2 n$ matrix

$$
\mathbf{H}_{\mathbf{q}}=\partial \mathbf{H} / \partial \mathbf{q}
$$

where the subscript denotes a derivative. Note that the Jacobian is defined in mathematical terms as the derivative of the transformation (Taylor and Mann 1972). In our formulation, the Jacobian matrix is the transformation between $\mathbf{H}$ (the constraint function) and $\mathbf{q}$ (the joint variables).

With the modified formulation including joint limits, the Jacobian is expanded as 


$$
\mathbf{H}_{\mathbf{q}}=\left[\begin{array}{c:c}
\boldsymbol{\xi}_{\mathbf{q}^{*}} & \mathbf{0} \\
\hdashline \mathbf{I} & \mathbf{q}_{\lambda}^{*}
\end{array}\right]
$$

where $\mathbf{q}_{\lambda}^{*}=\partial \mathbf{q}^{*} / \partial \lambda, \xi_{\mathbf{q}^{*}}=\partial \xi / \partial \mathbf{q}^{*}, \mathbf{0}$ is a $(3 \times n)$ zero matrix, $\mathbf{I}$ is the identity matrix, and

$$
\begin{gathered}
\boldsymbol{\xi}_{\mathbf{q}^{*}}=\left[\begin{array}{cccc}
x_{q_{1}} & x_{q_{2}} & \ldots & x_{q_{n}} \\
y_{q_{1}} & y_{q_{2}} & \ldots & y_{q_{n}} \\
z_{q_{1}} & z_{q_{2}} & \ldots & z_{q_{n}}
\end{array}\right] \\
\mathbf{q}_{\lambda}^{*}=\left[\begin{array}{cccc}
-\left(\left(q_{1}^{U}-q_{1}^{L}\right) / 2\right) \cos \lambda_{1} & 0 & \ldots & 0 \\
0 & -\left(\left(q_{2}^{U}-q_{2}^{L}\right) / 2\right) \cos \lambda_{2} & \ldots & 0 \\
0 & 0 & \ldots & 0 \\
0 & 0 & \ldots & -\left(\left(q_{n}^{U}-q_{n}^{L}\right) / 2\right) \cos \lambda_{n}
\end{array}\right]
\end{gathered}
$$

Because the Jacobian is not square (more than three degree of freedom), rank deficiency criteria were developed in the field of robotics (Abdel-Malek and Yeh 1997, Abdel-Malek, et al. 1997) and will be used to obtain all singular behavior of the Jacobian. Before addressing these criteria, however, it is important to show why the singularity of the Jacobian has a direct effect on identifying the workspace. A singularity (in the pure mathematical sense) is when the Jacobian has no inverse, i.e., a solution cannot be found. Indeed, boundaries to the workspace of limbs are associated with the inability to find solutions of Eq. (6) for any q (Abdel-Malek, et al. 1999).

Differentiation Eq. (1) with respect to time yields

$$
\dot{\mathbf{x}}=\xi_{\mathbf{q}} \dot{\mathbf{q}}^{*}
$$

where $\dot{\mathbf{q}}^{*}$ is the vector of joint velocities. Given a specified path trajectory velocity (i.e., given $\dot{\mathbf{x}}$ ), the calculation of $\dot{\mathbf{q}}^{*}$ requires computing an inverse of the Jacobian $\xi_{\mathbf{q}}$. For a singular Jacobian, it is not possible to compute the required velocities for such a path. It was also observed that such behavior is associated with boundaries to the workspace (e.g., when the arm is fully extended and cannot extend any further). 
We will use the idea of a singular Jacobian to identify all barriers inside and on the boundary of the workspace. Because the Jacobian is nonsquare, we define such barriers as a subset of the workspace at which the Jacobian of the constraint function of Eq. (7) is row rank deficient; i.e., barriers defined by $\partial W$ and characterized by

$$
\partial W \subset\left\{\operatorname{Rank} \mathbf{H}_{\mathbf{q}}(\mathbf{q})<k, \text { for some } \mathbf{q} \text { with } \mathbf{H}(\mathbf{q})=\mathbf{0}\right\}
$$

where $k$ is at least $(3+n-1)$. Because of the form of the Jacobian characterized by Eq. (12), three distinct conditions arise:

(1) Type I singularity sets: If no joints have reached their limits, the diagonal sub-matrix $\mathbf{q}_{\lambda}^{*}$ is full row rank. Therefore, the only possibility for $\mathbf{H}_{\mathbf{q}}$ to be row-rank deficient is when the block matrix $\xi_{q^{*}}$ is row rank deficient. Type I singularity set is defined as

$$
S^{(1)} \equiv\left\{\mathbf{p} \in \mathbf{q}^{*}: \operatorname{Rank}\left[\xi_{\mathbf{q}^{*}}\right]<3, \text { for some constant subset of } \mathbf{q}\right\}
$$

where $\mathbf{p}$ is within the specified joint limit constraints and may contain joints that are functions of others or constant values.

(2) Type II singularity sets: When certain joints reach their limits, e.g., $\partial \mathbf{q}^{\text {lim }}=\left[q_{i}^{\text {limit }}, q_{j}^{\text {limit }}, q_{k}^{\text {limit }}\right]^{T}$, the corresponding diagonal elements in the matrix $\mathbf{q}_{\lambda}^{*}$ will be equal to zero. Therefore, the corresponding $\Omega_{\mathbf{q}}$ is subjected to the rank-deficiency criterion, where $\mathbf{H}_{\mathbf{q}}$ will take on the following form

$$
\mathbf{H}_{\mathbf{q}} \sim\left[\begin{array}{ccccccc}
\boldsymbol{\xi}_{q_{1}} & \ldots & \boldsymbol{\xi}_{q_{i}} & \boldsymbol{\xi}_{q_{j}} & \boldsymbol{\xi}_{q_{k}} & \ldots & \boldsymbol{\xi}_{q_{n}} \\
0 & \ldots & 1 & 0 & 0 & \ldots & 0 \\
0 & \ldots & 0 & 1 & 0 & \ldots & 0 \\
0 & \ldots & 0 & 0 & 1 & \ldots & 0
\end{array}\right]
$$


and where the three columns pertaining to $\boldsymbol{\xi}_{q_{i}}, \boldsymbol{\xi}_{q_{j}}$, and $\boldsymbol{\xi}_{q_{k}}$ are removed such that the rank deficiency criteria are applied again. From the foregoing observation, the second type of singular sets are formulated. Define a new vector $\partial \mathbf{q}^{\text {limit }}=\left[q_{i}^{\text {limit }}, q_{j}^{\text {limit }}, q_{k}^{\text {limit }}\right]^{T}$ which is a sub-vector of $\mathbf{q}^{*}$ where $1 \leq \operatorname{dim}\left(\partial \mathbf{q}^{\text {limit }}\right) \leq(n-3)$

The type II singularity set is defined as

$$
S^{(2)} \equiv\left\{\mathbf{p}=\left[\hat{\mathbf{p}} \cap \partial \mathbf{q}^{\text {limit }}\right]: \operatorname{Rank}\left[\boldsymbol{\xi}_{\mathbf{q}^{*}}\left(\mathbf{w}, \partial \mathbf{q}^{\text {limit }}\right)\right]<3, \text { for some } \hat{\mathbf{p}} \in \mathbf{q}, \operatorname{dim}\left(\partial \mathbf{q}^{\text {limit }}\right) \leq(n-3)\right\}
$$

where $\hat{\mathbf{p}}$ is the singular set as a result of applying the rank deficiency criteria to Eq. (14).

(3) Type III singularity sets: are all sets that are composed of the combination of joints at their limits and is defined by:

$$
S^{(3)} \equiv\left\{\mathbf{p} \in \mathbf{R}^{(n-2)}: \mathbf{p} \equiv \partial \mathbf{q}^{\text {limit }}=\left[q_{i}^{\text {limit }}, q_{j}^{\text {limit }}, \ldots\right]\right\} ; \text { where } i \neq j
$$

Barriers are identified by substituting the sets $\mathbf{p}_{i}$ characterized by Eqs. $(13,15$, and 16) into the accessible set $\boldsymbol{\xi}\left(\mathbf{q}^{*}\right)$ yields the equation of a surface that can be readily shown.

Determining joint angles of a limb given a specific position and orientation is usually defined as the inverse kinematics problem in the robotics literature ( $\mathrm{Fu}$, et al. 1987). Motion from one configuration to another along a trajectory sometimes requires halting the motion and changing the inverse kinematics in order to proceed with the motion. An example of this occurs when attempting to reach a point located behind one's shoulder. Starting with one trajectory may become very uncomfortable because of joint limits, while trying another trajectory becomes simpler. Similarly, reaching a doorknob and turning sometimes is difficult to complete and requires orienting the initial hand configuration in a different posture. These barriers due to singular sets identified by Eqs. $(13,15,16)$ may admit motion only in one normal direction, and 
hence are called impediments to motion (Abdel-Malek, et al. 1999). In this case, the arm, for example, will not be able to cross such a barrier.

\section{Illustrative Example}

Before addressing a model of the arm with many degrees of freedom, we will illustrate the formulation to the arm while limited to planar motion (e.g., on the surface of a table). Consider the motion of the shoulder and the elbow where both joints are parallel (both revolute joints) and their axes perpendicular to the surface of the table as shown in Fig. 3. Note that the arm is modeled as two revolute joints also shown in Fig. 3. The point of interest is on the tip of the index finger.

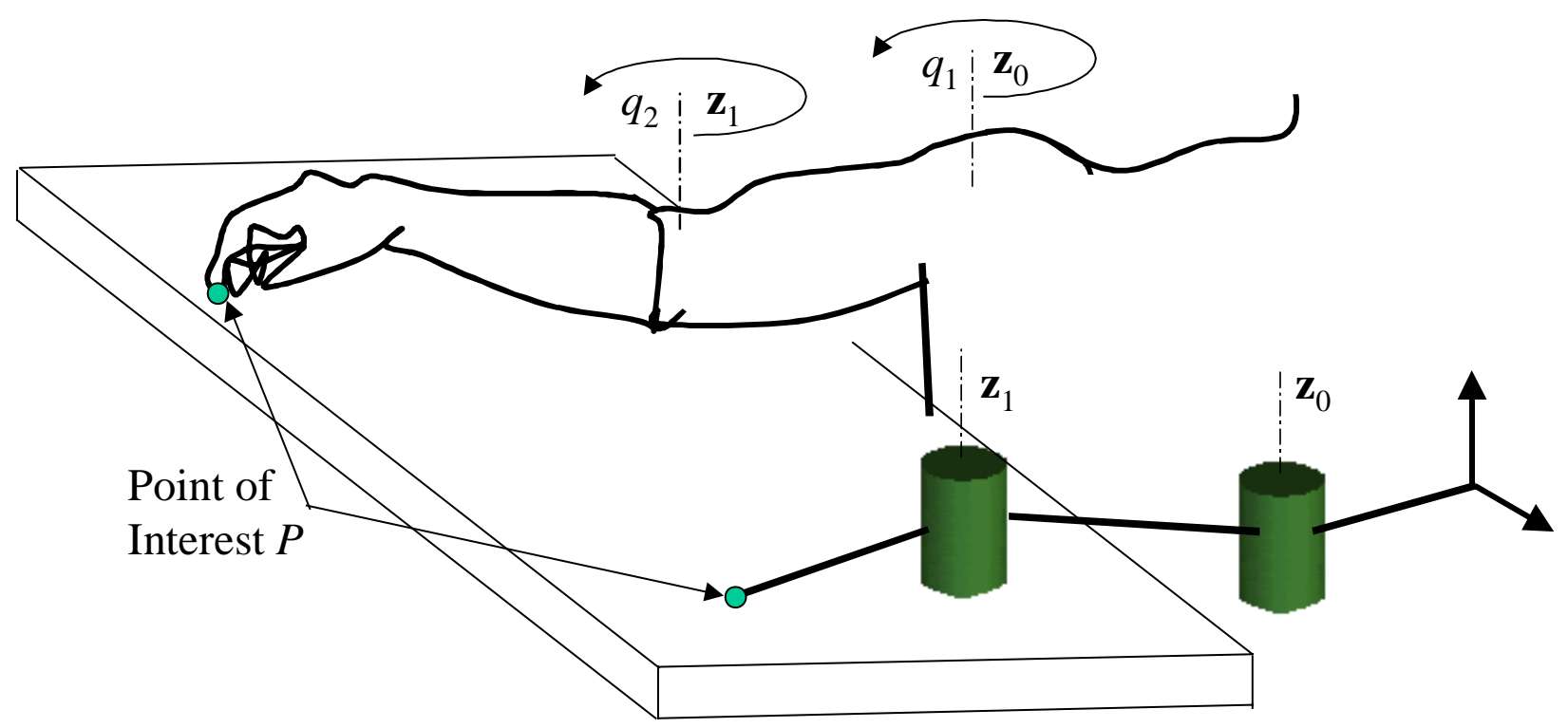

Fig. 3 Planar motion of the arm modeled as a two DOF system

Coordinates of the point of interest $P$ located at the tip of the index finger can be written as

$$
\boldsymbol{\xi}\left(\mathbf{q}^{*}\right)=\left[\begin{array}{c}
-10 \sin q_{1}-13 \sin \left(q_{1}+q_{2}\right) \\
10 \cos q_{1}+13 \cos \left(q_{1}+q_{2}\right)
\end{array}\right]
$$


with joint limits imposed as $-120^{\circ} \leq q_{1} \leq 70^{\circ}$ and $-5^{\circ} \leq q_{2} \leq 150^{\circ}$, where the tip of the index finger is positioned at $(5,0)$ with respect to the fourth coordinate system. These joint ranges of motion are converted into equality constraints as:

$$
\mathbf{q}^{*}(\boldsymbol{\lambda})=\left[\begin{array}{c}
(75-120) * \pi /(2 * 180)+(75+120) * \pi /(2 * 180) \sin \lambda_{1} \\
1.265+1.353 \sin \lambda_{2}
\end{array}\right]
$$

In order to compute the Jacobian singular behavior, we first calculate the Jacobian

$$
\boldsymbol{\xi}_{\mathbf{q}^{*}}=\left[\begin{array}{ll}
-10 \cos q_{1}-13 \cos \left(q_{1}+q_{2}\right) & -13 \cos \left(q_{1}+q_{2}\right) \\
-10 \sin q_{1}-13 \sin \left(q_{1}+q_{2}\right) & -13 \sin \left(q_{1}+q_{2}\right)
\end{array}\right]
$$

and

$$
\mathbf{q}_{\lambda}^{*}=\left[\begin{array}{cc}
-1.700 \cos \lambda_{1} & 0 \\
0 & -1.353 \cos \lambda_{2}
\end{array}\right]
$$

The singularity of the Jacobian is computed by setting the determinant of $\xi_{q^{*}}$ to zero (since it is a square matrix, otherwise determinant of all square sub-jacobians will be set to zero). The determinant of the Jacobian is

$$
\left|\xi_{\mathbf{q}^{*}}\right|=130 \sin q_{2}
$$

Setting Eq. (21) to zero yields a solution as $q_{2}=0$ (note that while $q_{2}=\pi$ is also a solution, it does not satisfy the joint range constraints). Similarly, applying the same criteria to $\mathbf{q}_{\lambda}^{*}$ yields the additional singularities (which are indeed the joint limits in this case). Substituting each joint limit into Eq. (17) yields a curve. For example, for $q_{2}=0$, the resulting equation is given by

$$
\xi^{(1)}\left(q_{1}, q_{2}=0\right)=\left[\begin{array}{c}
-23 \sin q_{1} \\
23 \cos q_{1}
\end{array}\right] ;\left(\text { at } q_{1}=q_{1}^{L}\right) \text { for } 120^{\circ} \leq q_{1} \leq 70^{\circ} \text { and shown in Fig. } 4
$$




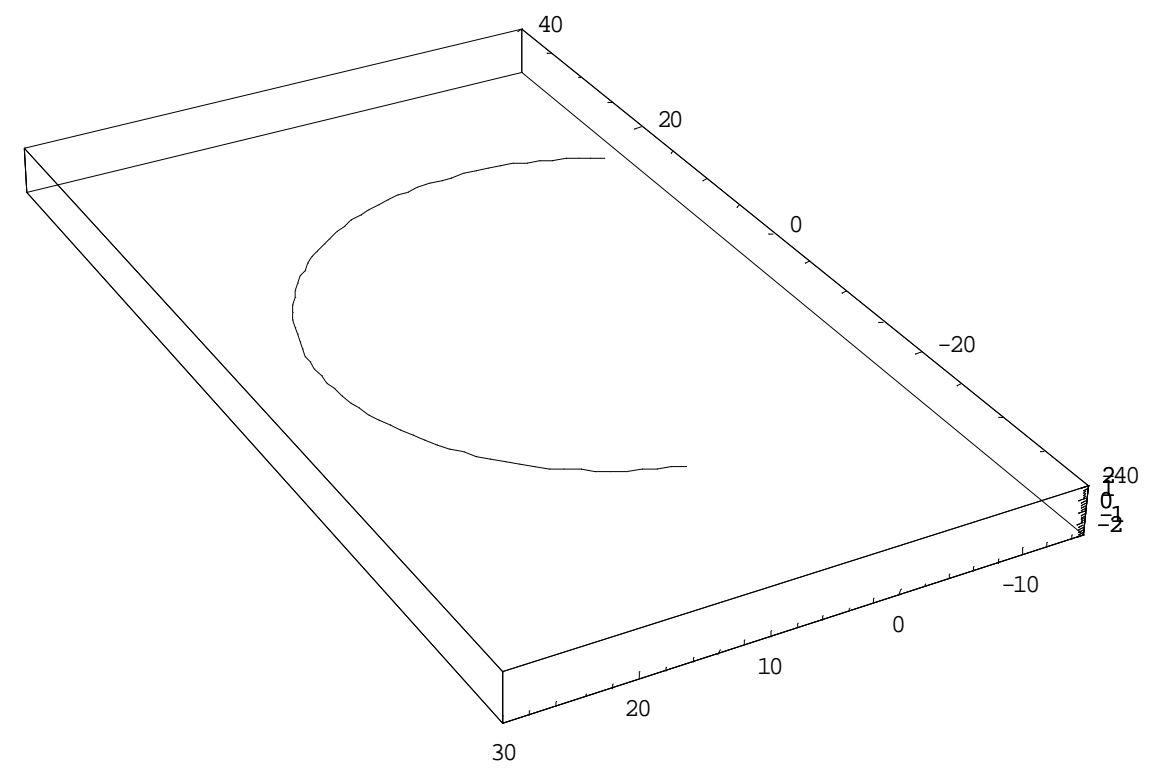

Fig. 3 Singular curve due to $q_{2}=0$

Similarly, substituting each singularity into $\xi\left(\mathbf{q}^{*}\right)$ yields a curve. The workspace is shown by plotting all curves in Fig. 4. 


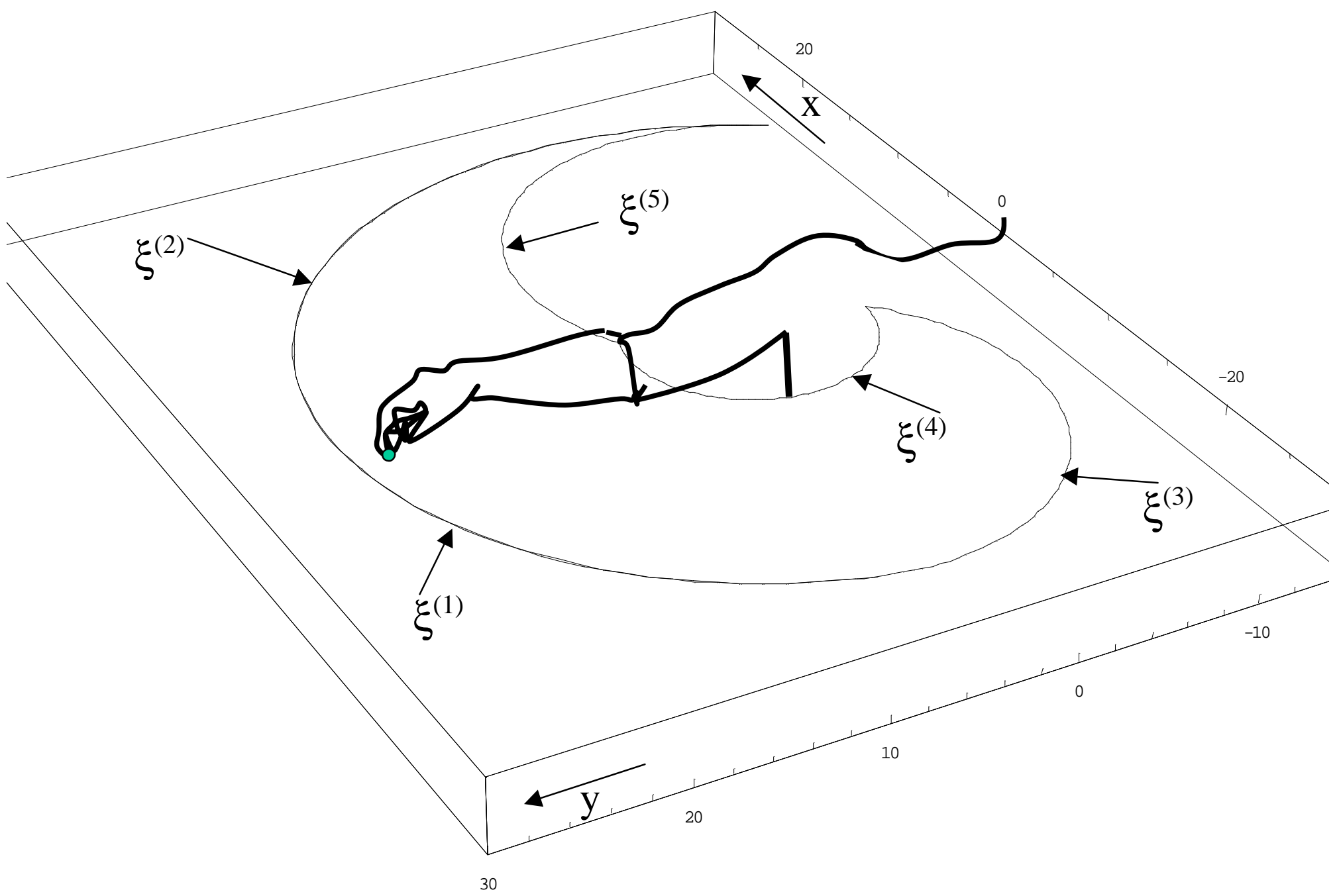

Fig. 4 The workspace envelope of the planar arm

\section{Forearm Workspace}

Consider the shoulder and forearm modeled as a 4-DOF system, where the spherical joint at the shoulder is modeled as three intersecting revolute joints and the elbow as a revolute joint. This is consistent with published results except that we have not considered the wrist joint (which is an additional 3 revolute joints) and we have limited the motion of the glenohumeral joint to spherical. Furthermore, we have modeled the spherical joint as three revolute joints intersecting at one point, a practice commonly made in modeling to represent spherical joints. It should be 
noted that the most difficult and the least successful modeling of a major articulating joint has been the shoulder because of the lack of appropriate biomechanical data as well as the anatomical complexity of the region. Figure 5 depicts the joint motions to be modeled where each joint is given an independent coordinate $q_{i}$ where the equivalent kinematic skeleton of the system is depicted with the z-axis located per the D-H representation method.

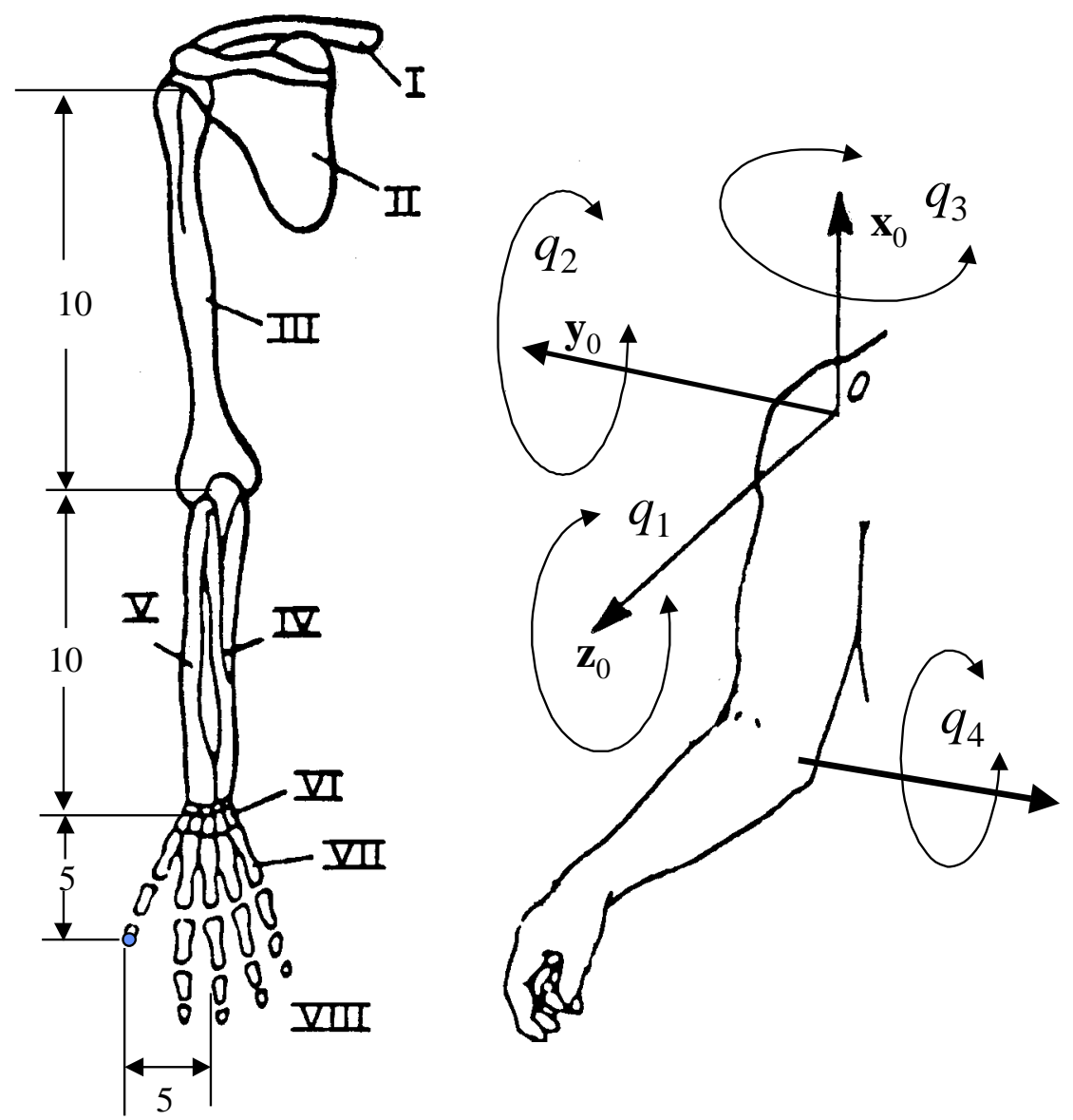

Fig. 5 Shoulder and arm and the corresponding degree of freedom

In the following analysis, a point on the tip of the thumb as shown in Fig. 6 will be tracked. The dimensions of the arm are also noted on the figure. In the field of kinematics, the motion of a spherical joint with three degrees of freedom can be modeled as three independent revolute joints having their axes intersecting at a single point as shown in Fig. 6. Note that the point on the 
thumb is shown located at the position ${ }^{4} \mathbf{v}=\left[\begin{array}{lll}5 & 0 & 5\end{array}\right]^{T}$ as resolved in the fourth coordinate frame.

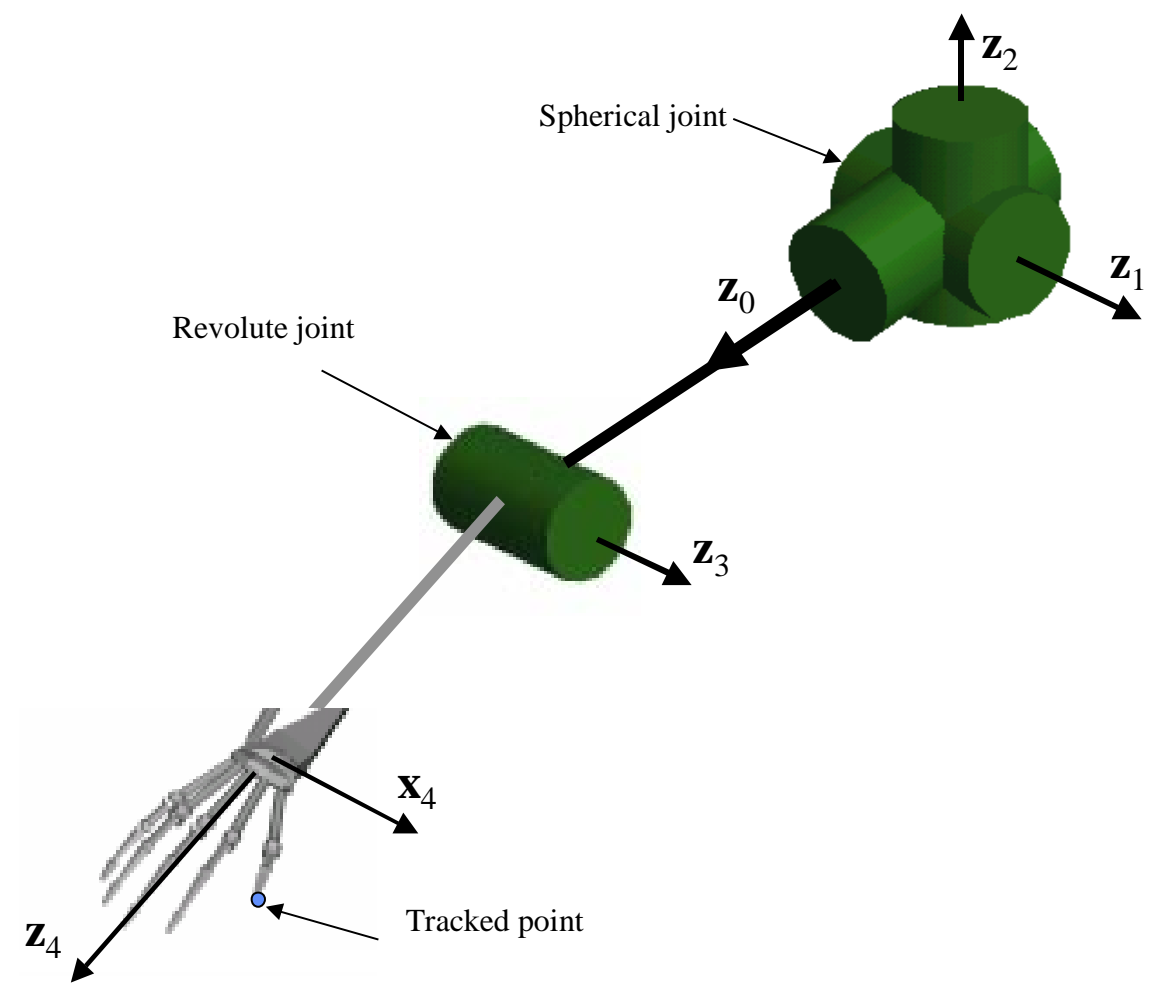

Fig. 6 Kinematic modeling of the forearm as a spherical joint and a revolute joint

It shoulde be noted that this model is limited only to the glenohumeral and not the scapulothoraic motion of the shoulder joint (i.e., the additional three translational degrees of freedom of the scapulothoraic are not taken into consideration).

In order to demonstrate our formulation, consider the following joint limits imposed on the model of Fig. 6: $-90^{\circ} \leq q 1 \leq 90,-110^{\circ} \leq q 2 \leq 120^{\circ},-90^{\circ} \leq q 3 \leq 90^{\circ}$, and $0^{\circ} \leq q 4 \leq 150^{\circ}$. Using the Denavit-Hartenberg representation method, the thumb position is given by Eq. (1) as $\xi(q)=\left[\begin{array}{c}5\{\cos q 3[\sin q 1-\cos q 1(4+5 \cos q 4) \sin q 2]+(4+5 \cos q 4) \sin q 1 \sin q 3+\cos q 1(\sin q 2 \sin q 3-5 \cos q 2 \sin q 4)\} \\ -5\{\cos q 1[\cos q 3+(4+5 \cos q 4) \sin q 3]+\sin q 1[\cos q 3(4+5 \cos q 4) \sin q 2-\sin q 2 \sin q 3+5 \cos q 2 \sin q 4]\} \\ 5\{\cos q 2[\cos q 3(4+5 \cos q 4)-\sin q 3]-5 \sin q 2 \sin q 4\}\end{array}\right]$ 
Rank deficiency criteria applied to the resulting $(3 \times 4)$ Jacobian matrix yields 40 singular sets that are listed in Appendix A. Note that most singular sets contain variables that use functions of other variables, i.e., coupled behavior. For example, substituting singular set $\mathbf{p}_{1}:\left(q_{1}=-\pi / 2\right.$ and $q_{2}=-110^{\circ}$ ) into Eq. (17) yields an exact closed form equation of a barrier as $\xi\left(\mathbf{u}_{1}\right)=\left[\begin{array}{c}-25 \sin q_{3} \cos q_{4}-5 \cos q_{3}-20 \sin q_{3} \\ -25 \sin (7 \pi / 18) \cos q_{3} \cos q_{4}-25 \cos (7 \pi / 18) \sin q_{4}+5 \sin (7 \pi / 18) \sin q_{3}-20 \sin (7 \pi / 18) \cos q_{3} \\ -25 \cos (7 \pi / 18) \cos q_{3} \cos q_{4}+25 \sin (7 \pi / 18) \sin q_{4}+5 \cos (7 \pi / 18) \sin q_{3}-20 \cos (7 \pi / 18) \cos q_{3}\end{array}\right]$

In order to explain the physical meaning of singular surfaces (barriers to motion), we use the skeleton model shown in Fig. 7 at the given singular configuration $\left(q_{1}=-\pi / 2\right.$ and $\left.q_{2}=-110\right)$, such that only $q_{3}$ and $q_{4}$ are allowed to vary. The surface shown in Fig. 7 is a geometric entity in space where the thumb is permitted to move. These surfaces may exist inside and on the boundary of the workspace. Some of these surfaces present impediments to motion because the arm will not admit motion in one of the normal directions (Abdel-Malek, et al. 1999).

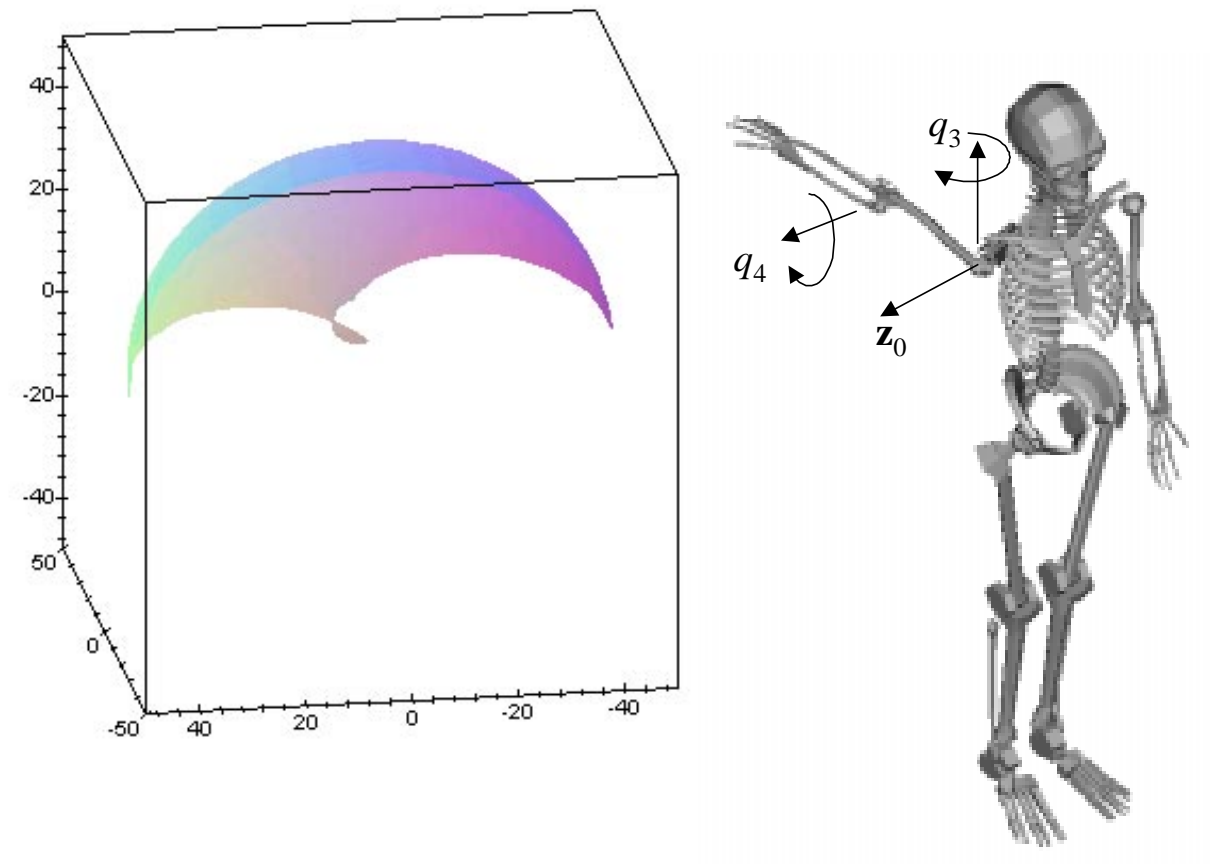


Fig. 7 Singular surface due to $\left(q_{1}=-\pi / 2\right.$ and $\left.q_{2}=-110\right)$ while allowing for $q_{3}$ and $q_{4}$ to vary

Similarly, for the singularity set at $q_{2}=-110$ and $q_{4}=-150$, and while varying $q_{1}$ and $q_{3}$, the singular surface and the corresponding arm configuration are shown in Fig. 8.

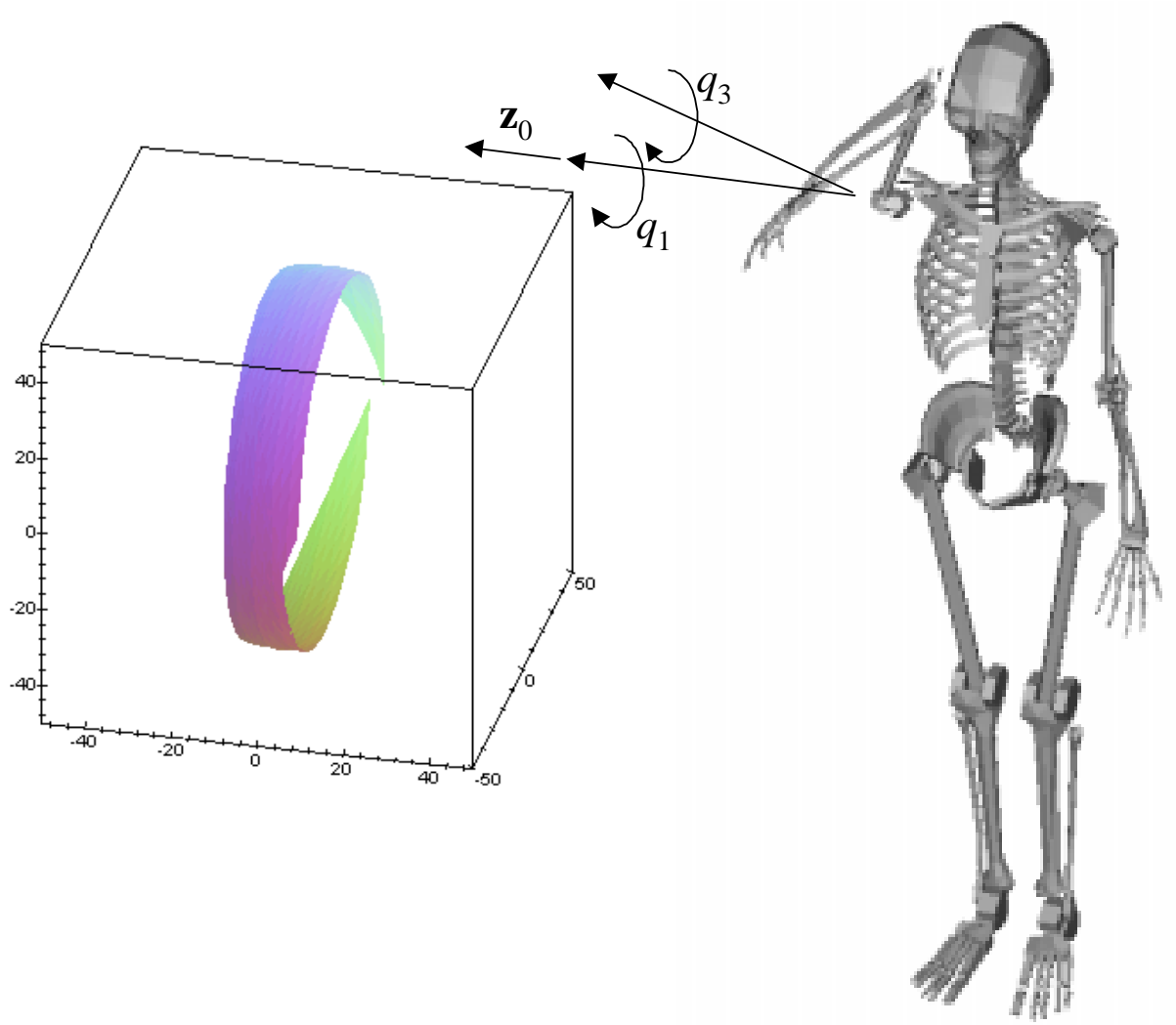

Fig. 8 The resulting surface due to a singularity set while allowing $q_{1}$ and $q_{3}$ to vary

Combining all 40 barriers yields the workspace of the forearm as shown in Fig. 9 (two crosssectional views are shown). 

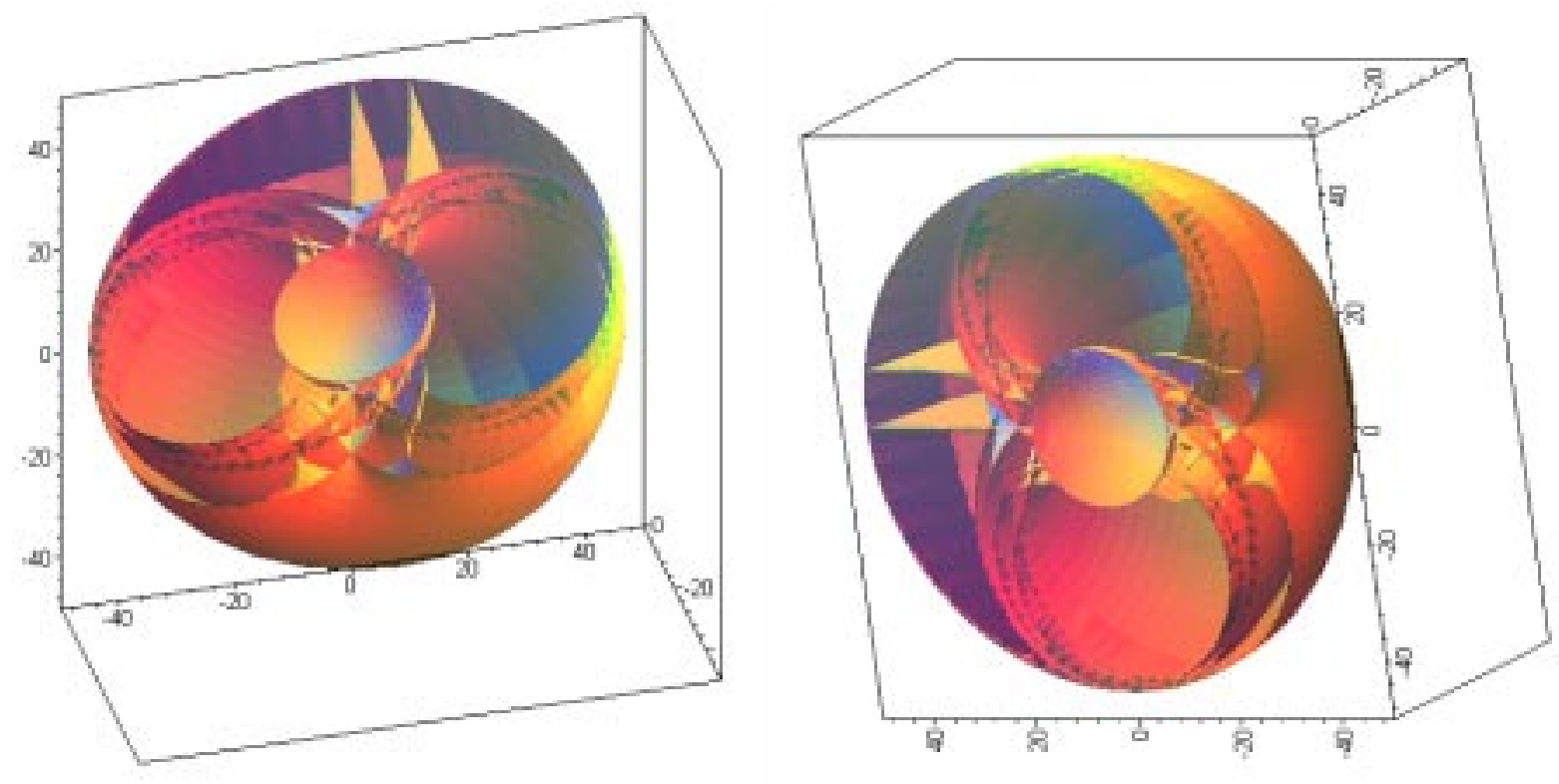

Fig. 9 Two cross-sectional views of the workspace of the forearm

Crossing a barrier: Some of the singular surfaces identified in Fig. 9 are impediments to motion. It was shown in recent work (Abdel-Malek, et al. 1999) that a configuration that does not admit motion in the direction normal to the surface (only in one direction), the surface is called a barrier. To explain a barrier, consider the configuration of the arm where the hand is positioned behind the shoulder. While this point is still in the workspace, no further motion is allowed. However, this same point can be reached with a different configuration of the arm, and yet the motion of the arm can continue beyond that point. Therefore, barriers are imaginary surfaces where a trajectory followed by the arm is interrupted because of the inability to find an admissible inverse kinematic solution through the surface. The workspace oriented with respect to the torso is shown in Fig. 10. 


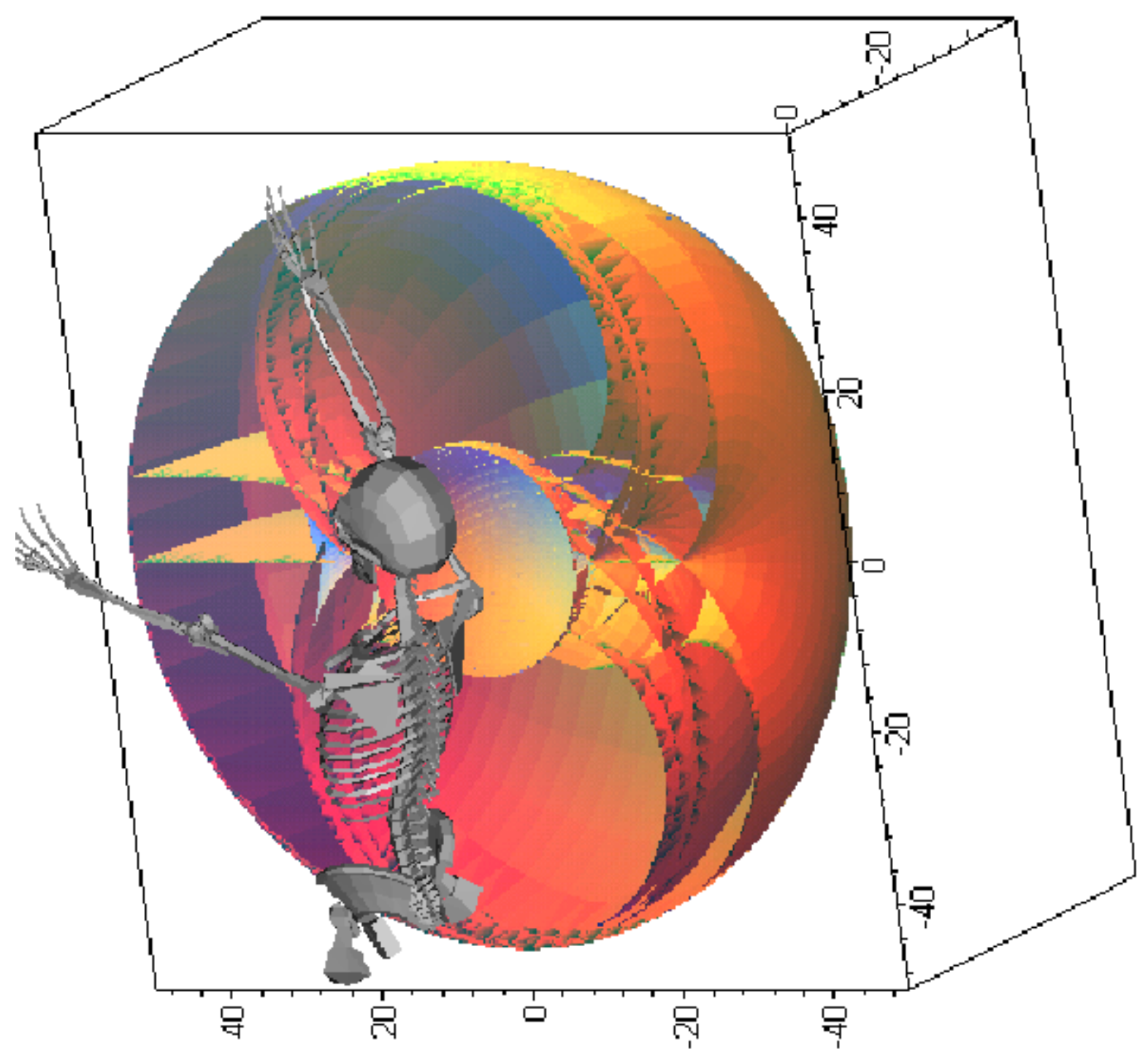

Fig. 10 Depicting the workspace and barriers with respect to the torso

\section{Finger Workspace}

Consider the workspace of a point located at the tip of the index finger as shown in Fig. 11a. The kinematic motion of the finger is modeled as four revolute joints, two of which intersect and shown in Fig. 11b. Limits for the finger joints are as follows: $0^{\circ} \leq q_{1} \leq 20^{\circ},-30^{\circ} \leq q_{2} \leq 50^{\circ}$, 
$0^{\circ} \leq q_{3} \leq 90^{\circ}$, and $0^{\circ} \leq q_{4} \leq 70^{\circ}$. The complete workspace (every point touched by $P$ ) is shown in Fig. 12.

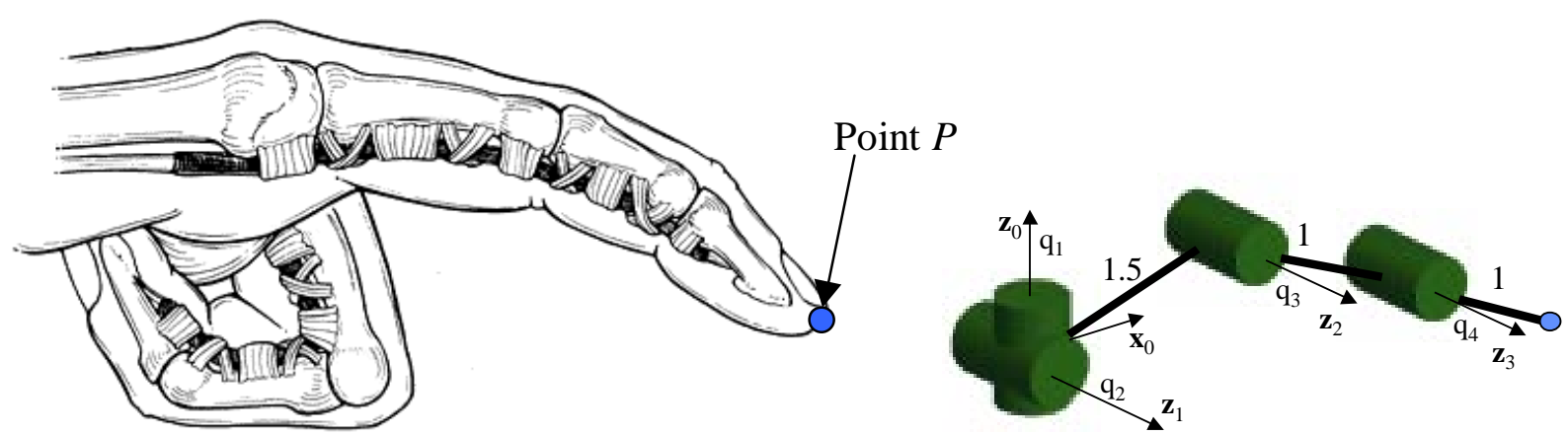

Fig. 11 (a) A schematic of a finger (b) Kinematic modeling of the finger as four revolute joints

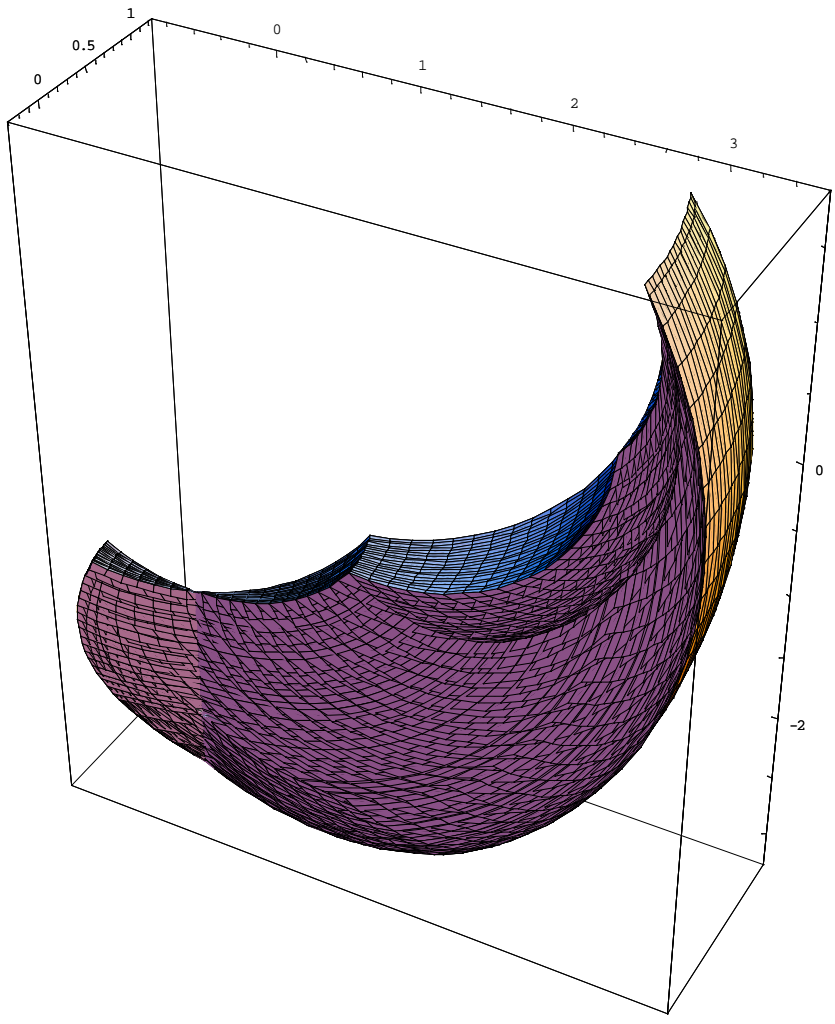

Fig. 12 Workspace of the finger

Wrist's Range of Motion (Quantifying the Workspace) 
As a direct application of this formulation, it is now possible to visualize the progress of a certain joint via workspace analysis. Note that measurement techniques and devices are well established. However, the range of motion is typically given in terms of a set of numerical joint angle values. The progress is difficult to monitor. We will show in this example that it is now possible to visualize the progress through a series of plots that depict the mobility of the joint (its workspace). Consider for example the wrist joint and hand shown in Fig. 13, where the wrist has been modeled as a 3-DOF system.
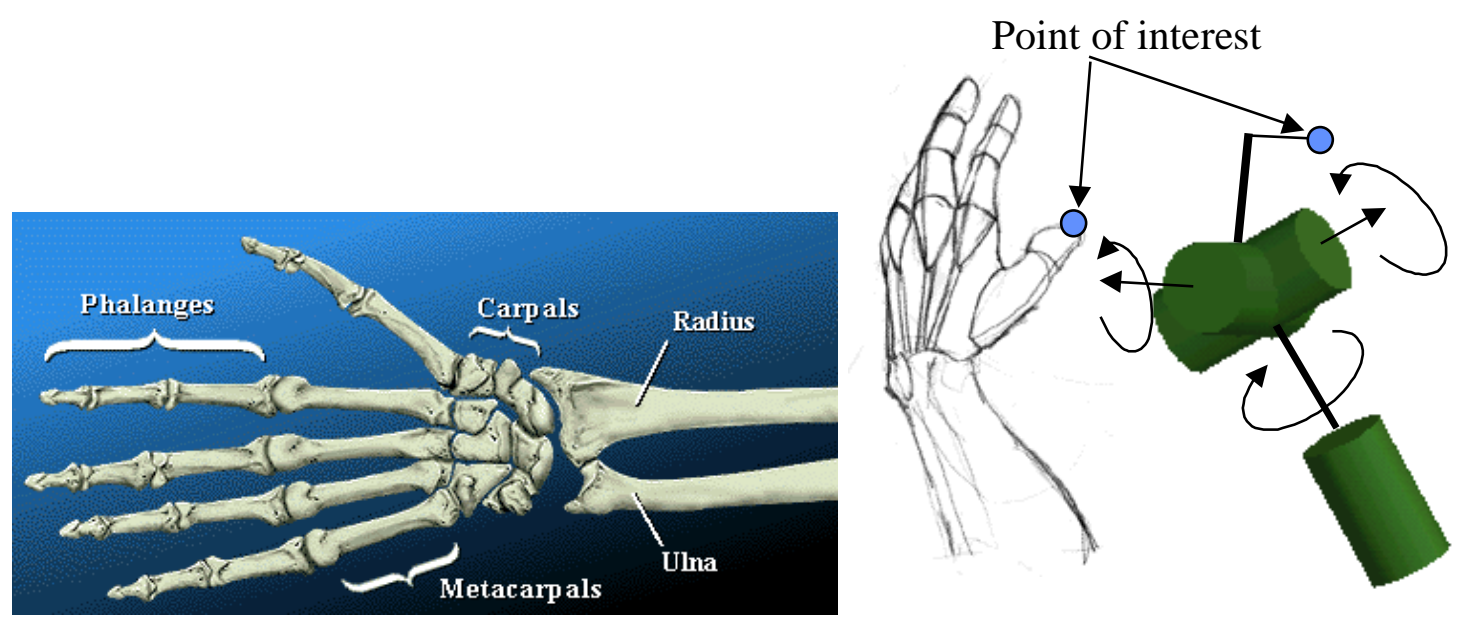

Fig. 13 (a) Wrist and hand (b) Modeling of the wrist joint

For an individual that has had a surgical procedure, the wrist joint motion may take weeks or months to return to normal or may be left with residual restrictions. Progress made, whether due to time alone or physical therapy, is measured using ranges of motion. Using the above formulation, not only visualization of the progress can effectively be made, but an accurate overall number can be used. Indeed, the surface area (or volume if the workspace is a volume), can be used to provide a good estimate. A normal joint range of motion for an adult is $-180^{\circ} \leq q_{1} \leq 45^{\circ},-70^{\circ} \leq q_{2} \leq 80^{\circ}$, and $-20^{\circ} \leq q_{3} \leq 40^{\circ}$, where the initial configuration of the hand is given as horizontal, thumb up, arm extended and away from the body. Using the above 
formulation, the resulting workspace is indeed a surface (a region of a spherical surface) as shown in Fig. 14.

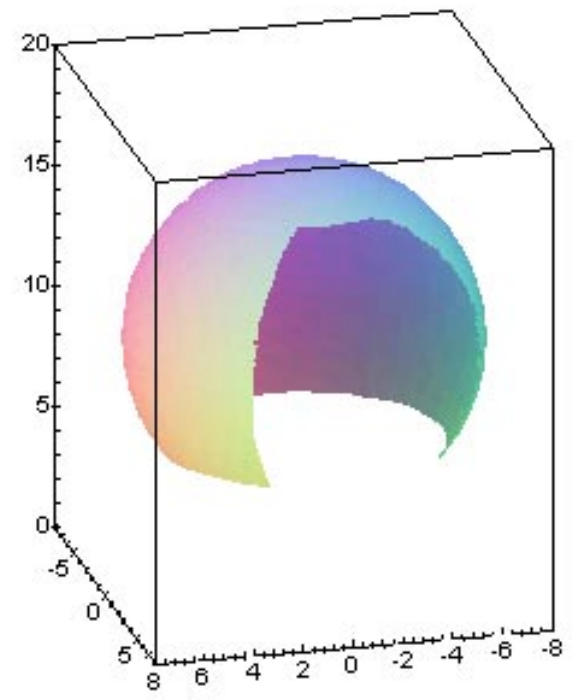

Fig. 14 The workspace of a point on the tip of the thumb with respect to the wrist

For a person who is undergoing physical therapy after a surgical operation, the functionality of the wrist may first be very limited. For example, immediately after the operation, the wrist joints may be limited to $-90^{\circ} \leq q_{1} \leq 10^{\circ},-30^{\circ} \leq q_{2} \leq 30^{\circ}$, and $-10^{\circ} \leq q_{3} \leq 20^{\circ}$, for which the workspace is shown in Fig. 15a. As the joint gains better mobility, the range of motion is increased and the progress is monitored by the visual workspace as shown in Figs. (15b-d), if an accurate measure is needed, the surface area obtained by an integration over the surface is performed. Note that this is only possible because of the ability to obtain equations of the boundary. 

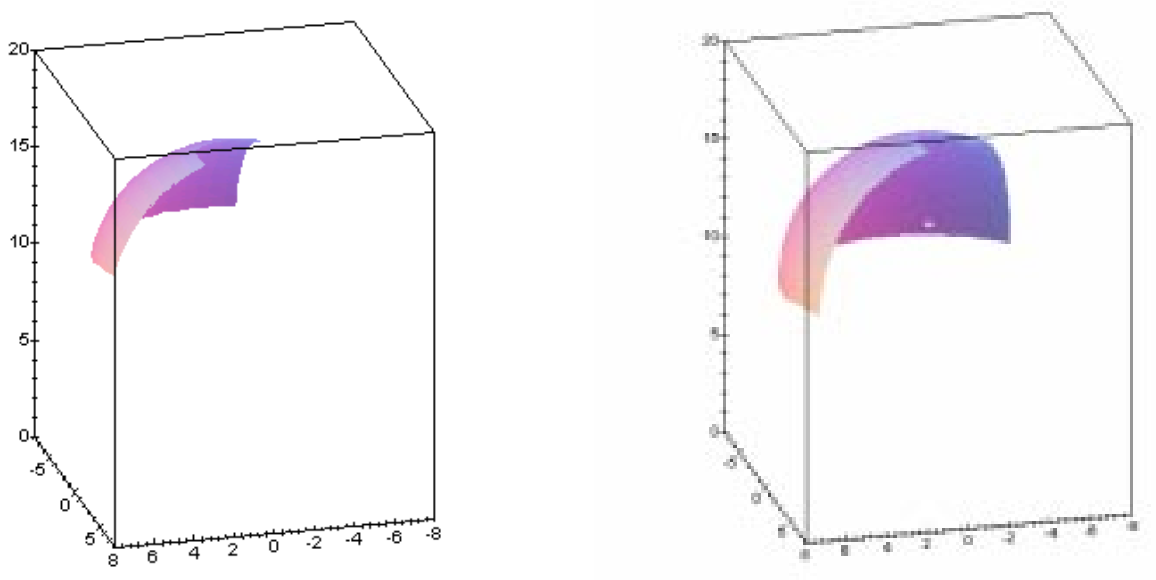

Fig. 15 (a) $-90^{\circ} \leq q_{1} \leq 10^{\circ},-30^{\circ} \leq q_{2} \leq 30^{\circ}$, and $-10^{\circ} \leq q_{3} \leq 20^{\circ}$, (b) $-120^{\circ} \leq q_{1} \leq 20^{\circ},-40^{\circ} \leq q_{2} \leq 45^{\circ}$, and $-13^{\circ} \leq q_{3} \leq 25^{\circ}$
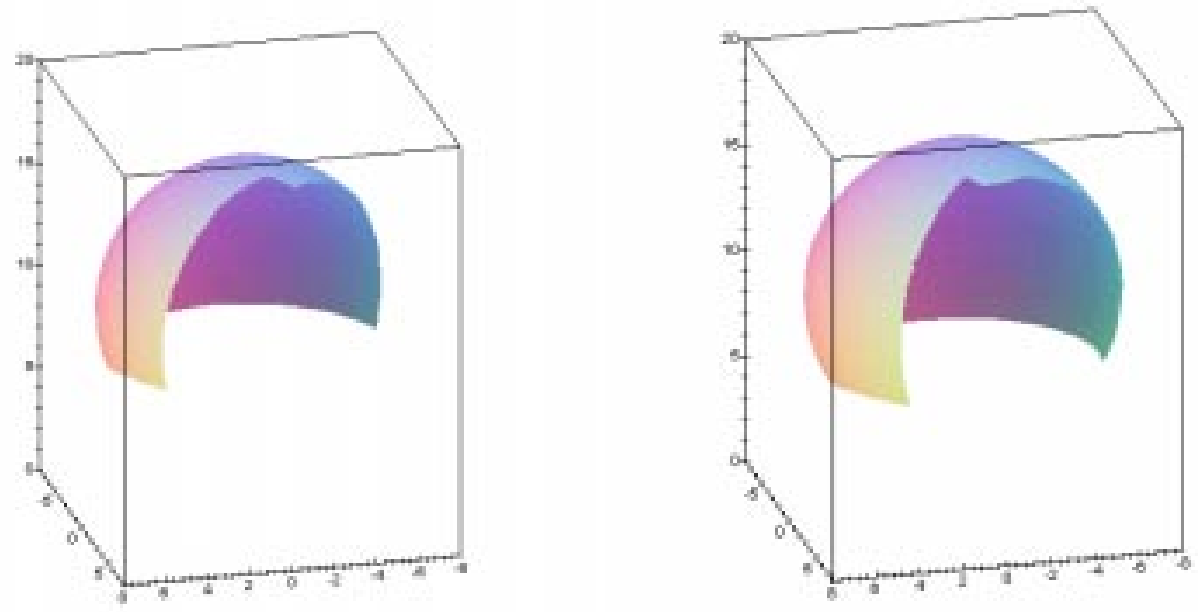

Fig. 15 (c) $-140^{\circ} \leq q_{1} \leq 30^{\circ},-50^{\circ} \leq q_{2} \leq 60^{\circ}$, and $-16^{\circ} \leq q_{3} \leq 32^{\circ}$ (d) $-160^{\circ} \leq q_{1} \leq 40^{\circ},-60^{\circ} \leq q_{2} \leq 70^{\circ}$, and $-18^{\circ} \leq q_{3} \leq 37^{\circ}$

\section{Conclusions}

A better understanding of the workspace of human limbs will aid researchers in better comprehending the central nervous system and the manipulation of motor commands. Furthermore, visualization of the exact workspace including barriers to motion where the limb is subjected to a singular configuration are also identified. These barriers play an important role in 
the identification of trajectories. Because of this analysis, visualization of the exact workspace is possible.

The present study has focused on geometric features of workspace and joint-space paths of threedimensional reaching movements. In this report, we have presented a rigorous formulation for modeling, analysis, and visualization of the workspace of human limbs. It was shown that barriers to trajectory control are explicitly identified in closed form and are based on the singularity of the Jacobian matrix. It was also shown using the proposed formulation that joint limits play an important role in distinguishing these barriers. While this is a first step towards investigating the workspace, only positioning (e.g., reacheability of the arm) is considered. Subsequent work will concentrate on a better understanding of the orientation workspace (e.g., wrist dexterity). Workspaces of the forearm and the finger were demonstrated. Because of this unique ability to generate the workspace boundary in closed form, it was shown that progress towards full mobility of a joint undergoing physical therapy, for example, is easily visualized and accurately quantified using a surface area calculation. The potential of this work to play an important role in the design for ergonomics and in providing a quantifiable measure for the functionality of joints is evident.

\section{References}

1. Abdel-Malek, K. and Yeh, H.J., 1997, "Geometric Representation of the Swept Volume Using Jacobian Rank-Deficiency Conditions," Computer Aided Design, Vol. 29, No. 6, pp. 457-468.

2. Abdel-Malek, K., Adkins, F., Yeh, H.J., and Haug, E.J., 1997, "On the Determination of Boundaries to Manipulator Workspaces," Robotics and Computer-Integrated Manufacturing, Vol. 13, No. 1, pp.63-72.

3. Abdel-Malek, K., Yeh, H.J., and Othman, S., 1999, "Swept Volumes, Void and Boundary Identification", Computer Aided Design, Vol. 30, Vol. 13, pp. 1009-1018. 
4. AMA, 1997, Guides to the Evaluation of Permanent Impairment. American Medical Association, Chicago, IL 1997.

5. Badler, N. I., "Real-time virtual humans", Proceedings of the 1997 5th Pacific Conference on Computer Graphics and Applications, Oct 13-16 19971997 Seoul, South Korea, p 4-13.

6. Boff, K. R. and Lincoln, J.E. (eds.), User's Guide: Engineering Data Compendium Human Perception and Performance. John Wiley and Sons, 1986.

7. Bruwer M, Cruse H, 1990, "A network model for the control of the movement of a redundant manipulator", Biol Cybern, 62(6):549-55.

8. Cruse H, Bruwer M, 1987, "The human arm as a redundant manipulator: the control of path and joint angles", Biol Cybern, 57(1-2):137-44.

9. Fu, K.S., Gonzalez, and Lee, C.S., 1987, Robotics: Control, Sensing, Vision, And Intelligence, McGraw-Hill, Inc., New York.

10. Johnson MW, Peckham PH, Bhadra N, Kilgore KL, Gazdik MM, Keith MW, Strojnik P, 1999, "Implantable transducer for two-degree of freedom joint angle sensing", IEEE Trans Rehabil Eng, 7(3):349-59.

11. Kalra, P. Beylot, P. Gingins, P. Magnenat-Thalmann, N., Volino, P. Hoffmeyer, P., Fasel, J., and Terrier, F., 1995, "Topological Modeling of Human Anatomy Using Medical Data" Proc. Computer Animation '95, IEEE Computer Society, pp. 172-180.

12. Kamper DG, Zev Rymer W, 1999, "Effects of geometric joint constraints on the selection of final arm posture during reaching: a simulation study", Exp Brain Res, 126(1):134-8.

13. Kawato M, Maeda Y, Uno Y, Suzuki R, 1990, "Trajectory formation of arm movement by cascade neural network model based on minimum torque-change criterion", Biol Cybern, 62(4):275-88.

14. Kawato M, Wolpert D, 1998, "Internal models for motor control”, Novartis Found Symp, 218:291-304; discussion 304-7.

15. Kirstukas SJ, Lewis JL, Erdman AG, 1992, “6R instrumented spatial linkages for anatomical joint motion measurement--Part 2: Calibration”, J Biomech Eng, 114(1):101-10.

16. Klein Breteler MD, Meulenbroek RG, Gielen SC, 1998, "Geometric features of workspace and joint-space paths of 3D reaching movements", Acta Psychol (Amst) 100(1-2):37-53.

17. Li, Shu; Xi, Z., "Measurement of functional arm reach envelopes for young Chinese males", Ergonomics, v 33 n 7 Jul 1990, pp. 967-978.

18. Maurel, W. and Thalmann D. "Human Upper Limb Modeling including Scapulo-Thoracic Constraint and Joint Sinus Cones" Computers \& Graphics, Vol. 24, No. 2, 2000 (in press).

19. Maurel, W. and Thalmann D. 1999, "A Case Study Analysis on Human Upper Limb Modelling for Dynamic Simulation" Computer Methods in Biomechanics and Biomechanical Engineering, Gordon and Breach Science Publishers, Vol. 1, No. 2, pp. 1-17.

20. Maurel, W., Thalmann, D. Hoffmeyer, P., Beylot, P.Gingins, P., Kalra, P., Magnenat, N. 1996 "A Biomechanical Musculoskeletal Model of Human Upper Limb for Dynamic Simulation" Proc. 7th Eurographics International Workshop on Computer Animation and Simulation, Aug 31-Sept 1, Poitiers, France, pp. 121-136.

21. Molenbroek, J. F.M., "Reach envelopes of older adults", Proceedings of the 1998 42nd Annual Meeting 'Human Factors and Ergonomics Society', Oct 5-Oct 91998 v 11998 Chicago, IL, pp. 166-170.

22. Paul, R.P., 1981, Robot Manipulators: Mathematics, programming, and Control. MIT Press, Cambridge, Massachusetts. 
23. Reinkensmeyer DJ, Dewald JP, Rymer WZ, 1999, “Guidance-based quantification of arm impairment following brain injury: a pilot study”, IEEE Trans Rehabil Eng, 7(1):1-11.

24. Richards, J. 1998, "The Measurement of Human Motion: A Comparison of Commercially Available Systems", Proceedings of the $5^{\text {th }}$ International Symposium on the 3-D analysis of Human Movement, Chabanacoque, Tennessee.

25. Schillings JJ, Meulenbroek RG, Thomassen AJ, 1998, "Functional properties of graphic workspace: assessment by means of a 3D geometric arm model", Acta Psychol (Amst) 100(1-2):97-115.

26. Suzuki M, Yamazaki Y, Mizuno N, Matsunami K, 1997, “Trajectory formation of the centerof-mass of the arm during reaching movements", Neuroscience, 76(2):597-610.

27. Uno Y, Kawato M, Suzuki R, 1989, "Formation and control of optimal trajectory in human multijoint arm movement. Minimum torque-change model", Biol Cybern, 61(2):89-101.

28. Van Thiel E, Meulenbroek RG, Hulstijn W, 1998, "Path curvature in workspace and in joint space: evidence for coexisting coordinative rules in aiming", Motor Control, 2(4):331-51.

29. $\mathrm{Yu} \mathrm{CH}$, Donaldson NN, 1999, "Measurement of posture by triangulation using potentiometers", Med Eng Phys, 21(4):259-63.

\section{Appendix A}

Singularity sets of the shoulder-forearm example:

$$
\begin{aligned}
& \mathbf{p}_{1}=\{q 1=-\pi / 2, q 2=-11 \pi / 18\}, \mathbf{p}_{2}=\{q 1=-\pi / 2, q 2=2 \pi / 3\}, \\
& \mathbf{p}_{3}=\{q 1=\pi / 2, q 2=-11 \pi / 18\}, \mathbf{p}_{4}=\{q 1=\pi / 2, q 2=2 \pi / 3\}, \\
& \mathbf{p}_{5}=\{q 1=-\pi / 2, q 3=-\pi / 2\}, \mathbf{p}_{6}=\{q 1=-\pi / 2, q 3=\pi / 2\}, \\
& \mathbf{p}_{7}=\{q 1=\pi / 2, q 3=-\pi / 2\}, \mathbf{p}_{8}=\{q 1=\pi / 2, q 3=\pi / 2\}, \\
& \mathbf{p}_{9}=\{q 2=-11 \pi / 18, q 3=\pi / 2\}, \mathbf{p}_{10}=\{q 2=-11 \pi / 18, q 3=-\pi / 2\}, \\
& \mathbf{p}_{11}=\{q 2=12 \pi / 18, q 3=-\pi / 2\}, \mathbf{p}_{12}=\{q 2=12 \pi / 18, q 3=\pi / 2\}
\end{aligned}
$$

The reduced rank deficiency singularity sets are as listed below.

$$
\begin{aligned}
& \mathbf{p}_{13}=\left\{q 1=-\pi / 2, q 3=-\operatorname{Arc} \cos \frac{1}{\sqrt{17+40 \cos q 4+25 \cos ^{2} q 4}}\right\}, \\
& \mathbf{p}_{14}=\left\{q 1=\pi / 2, q 3=-\operatorname{Arc} \cos \frac{1}{\sqrt{17+40 \cos q 4+25 \cos ^{2} q 4}}\right\}, \\
& \mathbf{p}_{15}=\left\{q 2=-11 \pi / 18, q 3=-\operatorname{Arc} \cos \frac{1}{\sqrt{17+40 \cos q 4+25 \cos ^{2} q 4}}\right\}, \\
& \mathbf{p}_{16}=\left\{q 2=2 \pi / 3, q 3=-\operatorname{Arc} \cos \frac{1}{\sqrt{17+40 \cos q 4+25 \cos ^{2} q 4}}\right\}, \\
& \mathbf{p}_{17}=\left\{q 1=-\pi / 2, q 3=\operatorname{Arccos} \frac{1}{\sqrt{17+40 \cos q 4+25 \cos ^{2} q 4}}\right\},
\end{aligned}
$$




$$
\begin{aligned}
& \mathbf{p}_{18}=\left\{q 1=\pi / 2, q 3=\operatorname{Arccos} \frac{1}{\sqrt{17+40 \cos q 4+25 \cos ^{2} q 4}}\right\}, \\
& \mathbf{p}_{19}=\left\{q 2=-11 \pi / 18, q 3=\operatorname{Arccos} \frac{1}{\sqrt{17+40 \cos q 4+25 \cos ^{2} q 4}}\right\}, \\
& \mathbf{p}_{20}=\left\{q 2=2 \pi / 3, q 3=\operatorname{Arccos} \frac{1}{\sqrt{17+40 \cos q 4+25 \cos ^{2} q^{4}}}\right\} \text {, } \\
& \mathbf{p}_{21}=\left\{q 1=-\pi / 2, q 3=-A r c \cos \frac{\sqrt{16+40 \cos q 4+25 \cos ^{2} q 4}}{\sqrt{17+40 \cos q 4+25 \cos ^{2} q 4}}\right\}, \\
& \mathbf{p}_{22}=\left\{q 1=\pi / 2, q 3=-\operatorname{Arc} \cos \frac{\sqrt{16+40 \cos q 4+25 \cos ^{2} q 4}}{\sqrt{17+40 \cos q 4+25 \cos ^{2} q^{4}}}\right\}, \\
& \mathbf{p}_{23}=\left\{q 2=-11 \pi / 18, q 3=-\operatorname{Arc} \cos \frac{\sqrt{16+40 \cos q 4+25 \cos ^{2} q 4}}{\sqrt{17+40 \cos q 4+25 \cos ^{2} q^{4}}}\right\}, \\
& \mathbf{p}_{24}=\left\{q 2=2 \pi / 3, q 3=-A r c \cos \frac{\sqrt{16+40 \cos q 4+25 \cos ^{2} q 4}}{\sqrt{17+40 \cos q 4+25 \cos ^{2} q 4}}\right\}, \\
& \mathbf{p}_{25}=\left\{q 1=-\pi / 2, q 3=\operatorname{Arccos} \frac{\sqrt{16+40 \cos q 4+25 \cos ^{2} q 4}}{\sqrt{17+40 \cos q 4+25 \cos ^{2} q 4}}\right\}, \\
& \mathbf{p}_{26}=\left\{q 1=\pi / 2, q 3=\operatorname{Arc} \cos \frac{\sqrt{16+40 \cos q 4+25 \cos ^{2} q^{4}}}{\sqrt{17+40 \cos q 4+25 \cos ^{2} q^{4}}}\right\}, \\
& \mathbf{p}_{27}=\left\{q 2=-11 \pi / 18, q 3=\operatorname{Arc} \cos \frac{\sqrt{16+40 \cos q 4+25 \cos ^{2} q 4}}{\sqrt{17+40 \cos q 4+25 \cos ^{2} q 4}}\right\}, \\
& \mathbf{p}_{28}=\left\{q 2=2 \pi / 3, q 3=\operatorname{Arccos} \frac{\sqrt{16+40 \cos q 4+25 \cos ^{2} q 4}}{\sqrt{17+40 \cos q 4+25 \cos ^{2} q 4}}\right\}, \\
& \mathbf{p}_{29}=\left\{q 1=-\pi / 2, q 2=-\operatorname{Arcsin} \frac{5 \sin q^{4}}{\sqrt{1+25 \sin ^{2} q^{4}}}\right\}, \\
& \mathbf{p}_{30}=\left\{q 1=-\pi / 2, q 2=-\operatorname{Arcsin} \frac{5 \sin q^{4}}{\sqrt{1+25 \sin ^{2} q^{4}}}\right\} \\
& \mathbf{p}_{31}=\left\{q 3=-\pi / 2, q 2=-\operatorname{Arcsin} \frac{5 \sin q^{4}}{\sqrt{1+25 \sin ^{2} q^{4}}}\right\}, \mathbf{p}_{32}=\left\{q 1=\pi / 2, q 2=\operatorname{Arcsin} \frac{5 \sin q 4}{\sqrt{1+25 \sin ^{2} q^{4}}}\right\} \text {, }
\end{aligned}
$$




$$
\begin{aligned}
& \mathbf{p}_{33}=\left\{q 1=-\pi / 2, q 2=\operatorname{Arcsin} \frac{5 \sin q 4}{\sqrt{1+25 \sin ^{2} q 4}}\right\}, \mathbf{p}_{34}=\left\{q 3=-\pi / 2, q 2=\operatorname{Arcsin} \frac{5 \sin q 4}{\sqrt{1+25 \sin ^{2} q 4}}\right\}, \\
& \mathbf{p}_{35}=\left\{q 3=\pi / 2, q 2=\arcsin \frac{5 \sin q 4}{\sqrt{1+25 \sin ^{2} q 4}}\right\}, \mathbf{p}_{36}=\left\{q 1=-\pi, q 4=\arcsin \frac{\tan q 2}{5}\right\}, \\
& \mathbf{p}_{37}=\left\{q 1=\pi / 2, q 4=\arcsin \frac{\tan q 2}{5}\right\}, \mathbf{p}_{38}=\left\{q 3=-\pi / 2, q 4=\arcsin \frac{\tan q 2}{5}\right\}, \\
& \mathbf{p}_{39}=\left\{q 3=\pi / 2, q 4=\arcsin \frac{\tan q 2}{5}\right\} .
\end{aligned}
$$

